\title{
Arginine/Asymmetric Dimethylarginine Ratio in Colorectal Surgery
}

\author{
Neli Ragina ${ }^{a}$, f, Gabrielle Davis ${ }^{\mathrm{b}}$, Michael Doorlyc, Kyle Cologne ${ }^{\mathrm{d}}$, \\ Anthony J. Senagore
}

\begin{abstract}
Background: Asymmetric dimethylarginine (ADMA) is a naturally occurring amino acid that inhibits the effects of nitric oxide synthetases, producing considerable effects on the vascular system. Recent studies have suggested that increased ADMA is a major contributor to endothelial dysfunction, resulting in significant effects on cardiovascular and renal function.
\end{abstract}

Methods: The study sample consisted of five male and 11 female patients scheduled for elective laparoscopic colorectal surgery; patients were between 41 and 77 years of age. Venous whole blood (8 - 10 $\mathrm{mL}$ ) was collected from patients prior to colorectal surgery, as well as on postoperative day 1 and 2. Arginine, citrulline, ADMA, and symmetric dimethylarginine levels were measured in collected blood using liquid chromatography coupled to mass spectrometry.

Results: Data from the amino-acid blood analysis demonstrated that the levels of L-citrulline and L-arginine decreased on postoperative days 1 , coinciding with an increase of ADMA levels. The overall result of these changes was a decrease in the arginine to ADMA ratio by $21 \%$ from the preoperative period to postoperative day $2(\mathrm{P}=0.02)$.

Conclusions: The data collected through this study demonstrate a significant increase in systemic ADMA levels following major laparoscopic colorectal surgery.

Keywords: ADMA; Citrulline; Nitric oxide; Surgery

\section{Introduction}

Asymmetric dimethylarginine (ADMA) is the most potent en-

\footnotetext{
Manuscript accepted for publication April 20, 2017

${ }^{a}$ Central Michigan University, College of Medicine, Mt. Pleasant, MI, USA

${ }^{\mathrm{b}}$ Stanford University School of Medicine, Palo Alto, CA, USA

${ }^{\mathrm{c}}$ Tuality Healthcare, Hillsboro Surgical Associates, Hillsboro, OR, USA

${ }^{\mathrm{d}}$ Keck School of Medicine of the University of Southern California, Los Angeles, CA, USA

eUniversity of Texas Medical Branch at Galveston, Galveston, TX, USA

${ }^{\mathrm{f} C o r r e s p o n d i n g ~ A u t h o r: ~ N e l i ~ R a g i n a, ~ C e n t r a l ~ M i c h i g a n ~ U n i v e r s i t y, ~ C o l l e g e ~ o f ~}$ Medicine, Mt. Pleasant, MI, USA. Email: raginane@gmail.com
}

doi: https://doi.org/10.14740/jocmr2988w dogenous inhibitor of all major isoforms of nitric oxide synthase (NOS). The elevation of ADMA has been implicated in disease pathology in a variety of conditions $[1,2]$. Elevation of ADMA has been particularly identified as an independent risk factor for adverse cardiovascular events and death potentially due to its negative effects on endothelial function [3-6]. Nijveldt et al recently identified ADMA elevation as the strongest predictor of negative outcome in critically ill patients in the intensive care unit [7]. Elevation of ADMA is the result of impaired metabolism by its major degrading enzyme, dimethylarginine dimethylaminohydrolase (DDAH) and/or impaired renal or liver excretion [8]. A rise in ADMA may therefore indicate impending liver and/or kidney failure, or more ominously failure of the endothelial system at large, all of which are responsible for eliminating ADMA.

Maas et al were the first to demonstrate that an incremental increase in preoperative plasma ADMA concentrations were associated with a 1.33 odds ratio of postoperative surgical complications [9]. These findings suggest that ADMA may be a novel tool in identifying individuals at increased perioperative risk [10]. A limitation in the authors' data was the absence of simultaneous assessment of arginine (ARG) levels to assess the relative impact of the ARG/ADMA ratio. The ratio of ARG/ADMA is an indirect measurement of nitric oxide functionality. ADMA degradation by DDAH leads to citrulline (CIT) production. Citrulline has been demonstrated to increase organ perfusion and improve outcomes in sepsis and pathological conditions [11]. The purpose of this study, therefore, was to provide the first assessment of perioperative changes in ARG, CIT, and the ARG/ADMA ratio as a result of surgical stress in a laparoscopic colectomy population.

\section{Methods}

The study is a descriptive, observational study. It was approved by the Institutional Review Board at the University of Southern California. A consecutive series of patients $(\mathrm{N}=16$, five males and 11 females) between 41 and 77 years of age that were scheduled for elective laparoscopic colorectal surgical procedures were enrolled in the study after providing informed consent. All of the patients received an oral mechanical and antibiotic bowel preparation prior to surgery. Surgical care improvement project guidelines as defined by the Joint Commission during the study period were implemented in all study participants. Postoperative care was managed using our institutions' enhanced recovery protocol, which has been published 
Table 1. Patients' Characteristics

\begin{tabular}{llll}
\hline Disease type & Age & Gender & Surgery \\
\hline Recurrent rectal cancer & 46 & F & Laparoscopic proctectomy w/coloanal anastomosis and loop ileostomy \\
Cecal cancer & 77 & F & Laparoscopic right hemicolectomy \\
Diverticulitis & 59 & F & Laparoscopic sigmoid colectomy \\
Right colon cancer & 71 & M & Laparoscopic right hemicolectomy \\
Diverticulitis & 54 & M & Laparoscopic sigmoid colectomy with release of splenic flexure \\
Rectal/sigmoid cancer & 62 & F & Laparoscopic anterior resection converted to open w/loop ileostomy \\
Cecal mass & 64 & F & Laparoscopic right hemicolectomy \\
Rectal prolapse & 41 & F & Laparoscopic Wells procedure for rectal prolapse \\
Carcinoid & 63 & F & Laparoscopic small bowel resection \\
Diverticulitis & 61 & M & Laparoscopic sigmoid colectomy \\
Rectal cancer & 66 & F & Laparoscopic low anterior resection w/loop ileostomy \\
Colovaginal fistula & 50 & F & Laparoscopic low anterior resection w/primary anastomosis \\
Colon cancer & 51 & M & Laparoscopic left colectomy w/takedown of splenic flexure \\
Colon cancer & 77 & F & Laparoscopic extended right hemicolectomy \\
Colon cancer & 70 & M & Laparoscopic Hartmann procedure \\
Ulcerative colitis with malignant degeneration & 50 & F & Laparoscopic total proctocolectomy w/ileoanal Jpouch and loop ileostomy \\
\hline
\end{tabular}

previously, but includes early resumption of diet and activity and minimization of narcotic analgesia [12]. Blood was drawn preoperatively, on postoperative day 1 (POD1), and POD2. Our standard of care was discharge at either the afternoon of POD2 or morning POD3; therefore, we terminated the testing period to accommodate our standard discharge practice. Shortterm complications were monitored and recorded up until the time of initial clinic follow-up.

\section{Isolation of serum from whole blood}

Eight to ten milliliters of venous whole blood was collected in red-topped tubes (Becton Dickinson (BD2)). The blood was allowed to clot by leaving it undisturbed at room temperature. The clot was removed by centrifuging at $1,000-2,000 \times \mathrm{g}$ for $10 \mathrm{~min}$ in a refrigerated centrifuge and the resulting supernatant (serum) was apportioned into $0.5 \mathrm{~mL}$ aliquots and stored at $-80{ }^{\circ} \mathrm{C}$.

\section{CIT, ARG, ADMA, and SDMA analysis}

Amino acids were measured by liquid chromatography coupled to mass spectrometry as previously described by Shin et al [13].

ARG, CIT, ADMA, and symmetrical dimethylarginine (SDMA) were purchased from Sigma. ARG, CIT, ADMA, and SDMA levels and ARG/ADMA ratio were assessed in serum at baseline (pre-operatively) and POD1 and POD2. Serum samples were diluted with water 100 times for ARG, CIT, ADMA and SDMA analysis. The analysis of ARG, CIT, ADMA and SDMA was performed by reverse-phase high performance liquid chromatography (HPLC) on-line to a TSQ Quantum Ac- cess MAX triple stage quadrupole mass spectrometer. Quantification was performed via single reaction monitoring (SRM) using a fragment ion unique to ARG, CIT, ADMA and SDMA, respectively.

\section{Statistical analysis}

All data were presented as mean and standard deviation (SD) from the mean or ratios as defined by the data. Statistical analysis consisted of ANOVA and $t$-test with $\mathrm{P}<0.05$ considered as statistically significant.

\section{Results}

Table 1 lists the demographics of our patient population. All patients underwent laparoscopic colorectal procedures for cancer, complications of diverticulitis and rectal prolapse. Our data demonstrated a statistically significant increase of the ADMA levels on POD2 (22 ng/mL (SD: 18 - 26) vs. $28 \mathrm{ng} /$ $\mathrm{mL}$ (SD: 22 - 34); $\mathrm{P}=0.02$ ) (Fig. 1). The net result of these changes was a decrease in the ARG/ADMA ratio by $21 \%$ from the preoperative period to POD2 $(\mathrm{P}=0.02)$ (Fig. 1). The ARG/ ADMA drop in our study was apparently related only to the surgical stress response as none of the patients developed clinical infections. No significant changes were noted in the CIT level by POD2.

\section{Discussion}

These are the first preliminary data to demonstrate a clear and 


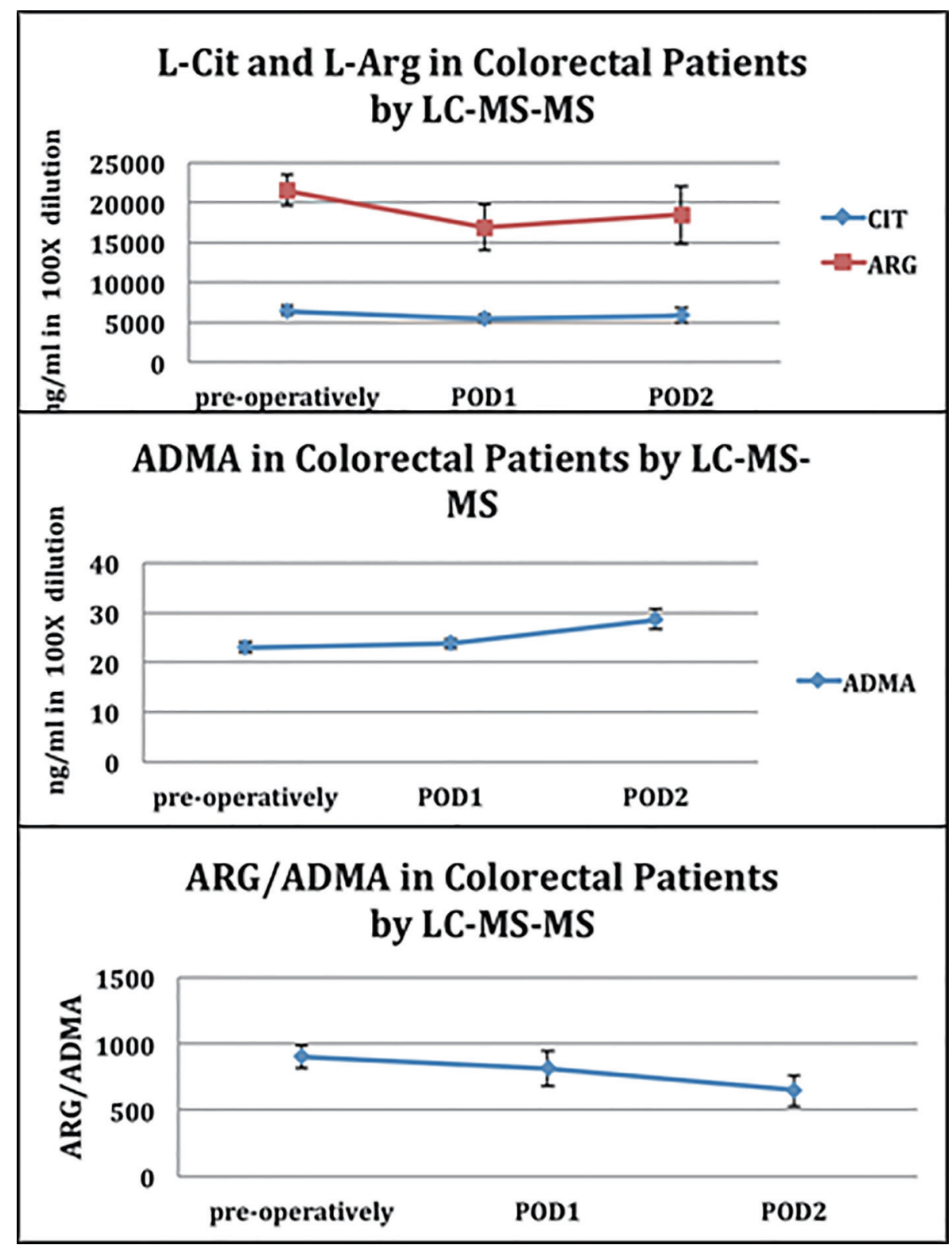

Figure 1. L-citrulline (L-Cit), L-arginine (L-Arg), ADMA and ARG/ADMA ratio in patients undergoing colon and rectal surgery.

significant rise in systemic ADMA levels after laparoscopic colorectal surgery, even in the absence of surgical complications. There was a subsequent reduction in the ARG/ADMA ratio that was statistically significant that indicates a decrease in nitric oxide functionality. The clinical significance of this remains uncertain; however, it does demonstrate that surgical stress leads to changes of these parameters.

Long-term inhibition of endothelial NOS (eNOS) by ADMA has been associated with disease progression and increased morbidity and mortality [14-16]. It has also been postulated that acute reduction in inducible NOS (iNOS) during episodes of acute inflammation such as in sepsis may be desirable, yet this theory is questionable [17-19]. The precise role of ADMA alone serves as either a physiologic brake to iNOS activity, or alternatively in combination with low L-arginine plasma levels decreased the producing decreased systemic vascular resistance and impaired the central organ blood flow leading to multisystem organ failure [20-22]. There is a significant body of literature confirming that trauma results in a reduction in circulating $A R G$ due to the activation of arginase. In addition, ARG reduction is directly correlated to injury severity $[22,23]$. Although the liver is the predominant source of constitutively active arginase activity, which increases in response to surgical trauma, both the endothelium and neutrophil degranulation may be additional sources of circulating arginase in the trauma setting [24].

Interestingly, these data are the first to assess the interrelationship of ARG and ADMA in a major surgical population managed within an enhanced recovery program. This ratio has never been investigated in clinical trials of NOS inhibitors or 
as part of immunonutrition studies. In fact, this relationship has been advocated as an important contributor to the "ARG paradox" describing the general failure of ARG supplementation in improving clinical outcomes in various treatment trials.

The data in this study will require confirmation in a larger patient population, and correlated with other clinic outcome parameters to determine if ADMA fluctuations could be used as a marker of surgical outcomes. It will also be important to determine if inhibiting ADMA by its inhibitor, DDAH, could be used to combat the deleterious effects of ADMA. In our data, the elevation in ADMA and the decrease in the ARG/ ADMA ratio were still declining at $48 \mathrm{~h}$ post-surgery and therefore we cannot comment on the time required for resolution. These limited data make it difficult to draw any conclusions regarding the relative impact of a preoperative ARG or CIT supplementation. Ultimately, data from an extended perioperative time frame will be required to assess the impact of alterations in ADMA levels and the ARG/ADMA ratio on NO functionality during the various phases of the postoperative period: 1) the acute phase response; 2) during the development of complications; or 3) during the uncomplicated healing phase after surgery. However, the data are supportive of the potential role for the ARG/ADMA ratio as an important surrogate marker in perioperative responses to surgical stress and recovery. These data also suggest the feasibility of using L-citrulline as possible regulator of ADMA levels and ARG/ADMA ratio, via upregulation of L-arginine synthesis, in patients during the postoperative phase. A larger study with longer follow-up appears well warranted to more fully understand the variation in clinical responses of the ARG/ADMA ratio and clinical outcome.

\section{Disclosures}

This is a research involving human subjects and is performed under an approved IRB and signed informed consent by the participants.

\section{Conflicts of Interest}

The authors disclose that they do not have any potential conflicts of interest.

\section{References}

1. Cooke JP. Asymmetric dimethylarginine: The Uber marker? Circulation. 2004;109:1813-1818.

2. Vallance P, Leiper J. Cardiovascular biology of the asymmetric dimethylarginine:dimethylarginine dimethylaminohydrolase pathway. Arterioscler Thromb Vasc Biol. 2004;24(6):1023-1030.

3. Valkonen VP, Paiva H, Salonen JT, Lakka TA, Lehtimaki T, Laakso J, Laaksonen R. Risk of acute coronary events and serum concentration of asymmetrical dimethylarginine. Lancet. 2001;358(9299):2127-2128.
4. Zoccali C, Bode-Boger S, Mallamaci F, Benedetto F, Tripepi G, Malatino L, Cataliotti A, et al. Plasma concentration of asymmetrical dimethylarginine and mortality in patients with end-stage renal disease: a prospective study. Lancet. 2001;358(9299):2113-2117.

5. Lu TM, Ding YA, Lin SJ, Lee WS, Tai HC. Plasma levels of asymmetrical dimethylarginine and adverse cardiovascular events after percutaneous coronary intervention. Eur Heart J. 2003;24(21):1912-1919.

6. Schnabel R, Blankenberg S, Lubos E, Lackner KJ, Rupprecht HJ, Espinola-Klein C, Jachmann N, et al. Asymmetric dimethylarginine and the risk of cardiovascular events and death in patients with coronary artery disease: results from the AtheroGene Study. Circ Res. 2005;97(5):e53-59.

7. Nijveldt RJ, Teerlink T, Van Der Hoven B, Siroen MP, Kuik DJ, Rauwerda JA, van Leeuwen PA. Asymmetrical dimethylarginine (ADMA) in critically ill patients: high plasma ADMA concentration is an independent risk factor of ICU mortality. Clin Nutr. 2003;22(1):23-30.

8. Murphy RB, Tommasi S, Lewis BC, Mangoni AA. Inhibitors of the Hydrolytic Enzyme Dimethylarginine Dimethylaminohydrolase (DDAH): Discovery, Synthesis and Development. Molecules. 2016;21(5).

9. Maas R, Dentz L, Schwedhelm E, Thoms W, Kuss O, Hiltmeyer N, Haddad M, et al. Elevated plasma concentrations of the endogenous nitric oxide synthase inhibitor asymmetric dimethylarginine predict adverse events in patients undergoing noncardiac surgery. Crit Care Med. 2007;35(8):1876-1881.

10. Brinkmann SJ, de Boer MC, Buijs N, van Leeuwen PA. Asymmetric dimethylarginine and critical illness. Curr Opin Clin Nutr Metab Care. 2014;17(1):90-97.

11. Wijnands KA, Vink H, Briede JJ, van Faassen EE, Lamers WH, Buurman WA, Poeze M. Citrulline a more suitable substrate than arginine to restore NO production and the microcirculation during endotoxemia. PLoS One. 2012;7(5):e37439.

12. Rona K, Choi J, Sigle G, Kidd S, Ault G, Senagore AJ. Enhanced recovery protocol: implementation at a county institution with limited resources. Am Surg. 2012;78(10):1041-1044.

13. Shin S, Fung SM, Mohan S, Fung HL. Simultaneous bioanalysis of L-arginine, L-citrulline, and dimethylarginines by LC-MS/MS. J Chromatogr B Analyt Technol Biomed Life Sci. 2011;879(7-8):467-474.

14. Schwedhelm E, Tan-Andresen J, Maas R, Riederer U, Schulze F, Boger RH. Liquid chromatography-tandem mass spectrometry method for the analysis of asymmetric dimethylarginine in human plasma. Clin Chem. 2005;51(7):1268-1271.

15. Sydow K, Schwedhelm E, Arakawa N, Bode-Boger SM, Tsikas D, Hornig B, Frolich JC, et al. ADMA and oxidative stress are responsible for endothelial dysfunction in hyperhomocyst(e)inemia: effects of L-arginine and B vitamins. Cardiovasc Res. 2003;57(1):244-252.

16. Vallance P, Leone A, Calver A, Collier J, Moncada S. Accumulation of an endogenous inhibitor of nitric oxide synthesis in chronic renal failure. Lancet. 
1992;339(8793):572-575.

17. Kilbourn R. Nitric oxide synthase inhibitors - a mechanism-based treatment of septic shock. Crit Care Med. 1999;27(5):857-858.

18. Lopez A, Lorente JA, Steingrub J, Bakker J, McLuckie A, Willatts S, Brockway M, et al. Multiple-center, randomized, placebo-controlled, double-blind study of the nitric oxide synthase inhibitor 546C88: effect on survival in patients with septic shock. Crit Care Med. 2004;32(1):2130 .

19. Cobb JP. Use of nitric oxide synthase inhibitors to treat septic shock: the light has changed from yellow to red. Crit Care Med. 1999;27(5):855-856.

20. Achan V, Broadhead M, Malaki M, Whitley G, Leiper J, MacAllister R, Vallance P. Asymmetric dimethylarginine causes hypertension and cardiac dysfunction in humans and is actively metabolized by dimethylarginine dimethylaminohydrolase. Arterioscler Thromb Vasc Biol. 2003;23(8):1455-1459.

21. Boger RH, Bode-Boger SM, Szuba A, Tsao PS, Chan JR, Tangphao O, Blaschke TF, et al. Asymmetric dimethylarginine (ADMA): a novel risk factor for endothelial dysfunction: its role in hypercholesterolemia. Circulation. 1998;98(18):1842-1847.

22. Maas R. Pharmacotherapies and their influence on asymmetric dimethylargine (ADMA). Vasc Med. 2005;10(Suppl 1):S49-57.

23. Ochoa JB, Bernard AC, O'Brien WE, Griffen MM, Maley ME, Rockich AK, Tsuei BJ, et al. Arginase I expression and activity in human mononuclear cells after injury. Ann Surg. 2001;233(3):393-399.

24. Pribis JP, Zhu X, Vodovotz Y, Ochoa JB. Systemic arginine depletion after a murine model of surgery or trauma. JPEN J Parenter Enteral Nutr. 2012;36(1):53-59. 